\title{
Efficacy of Working Memory training on Craving and Cognitive Functioning of Opiate Users
}

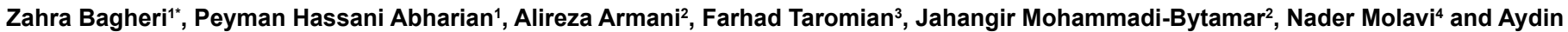
Moradi $^{5}$

${ }^{1}$ Institute for Cognitive Science Studies, Brain and Cognition Clinic, Tehran, Iran

${ }^{2}$ Zanjan University of Medical Sciences, Zanjan, Iran

${ }^{3}$ University of Social Welfare and Rehabilitation Sciences, Tehran, Iran

${ }^{4}$ Faculty of Medicine, Kashan University of Medical Sciences, Kashan, Iran

${ }^{5}$ Technical Responsibility of Raha Drug Abuse Treatment Clinic, Zanjan, Iran

\begin{abstract}
Objective: The aim of this study was to examine the efficacy of computer-assisted cognitive rehabilitation treatment (CACRT), working memory training, as a complementary treatment of opiate craving in-patients with substance use disorder.

Methods: Thirty opiate users receiving methadone maintenance treatment (MMT) were randomly allocated to two groups: MMT plus CACRT for the experimental group and standard methadone treatment only for the control group. In the experimental group, participants received 15 sessions of CACRT. All participants in both groups were assessed on measures of craving, impulsivity, delay discounting, and risky decision making at baseline and after treatment completion.

Results: Participants in the experimental group showed significant working memory improvements as well as craving reductions, accompanied by a positive effect on impulsivity and delay discounting. However, there was no significant difference in terms of risky decision-making between participants from the experimental group and participants from the control group.
\end{abstract}

Conclusion: These results suggest improvement of working memory has positive effects on impulsivity, delay discounting ability, and craving reduction.

Keywords: Working memory; Craving; Opiate users; Cognitive rehabilitation; Delay discounting

\section{Introduction}

Addiction is a pathological, behavioral, and physical dependence on one or more drugs. Its hallmarks are drug-seeking behaviors and pathological consumption patterns which lead to the occurrence of withdrawal syndrome in the absence of the drug(s) [1,2].

In this class of disorders, craving and voracity are inevitable, compose the core of the problem, and are the primary reasons for relapse [3]. Voracity, as a personal experience and a multidimensional phenomenon, is mixed with desire and passion, and its ultimate goal is to achieve a pleasant feeling or to overcome an undesired sensation. It covers a wide range of phenomena, including expectations of drugs reinforcing effects, intention to use drugs, and proclivity to drugs [4]. This feeling may last a few hours after the start of the treatment up to days and months after it has ended. The frequency and intensity of this feeling begin to decline over time but rarely disappears completely [5].Numerous studies have reported that chronic use of substances is related to cognitive defects and a significant sharp decline in cognitive functions such as decision-making, impulsivity, and working memory [6,7]. Unfortunately, these defects are not reversible [8,9].

Working memory, as a subjective system, plays a major role in learning and performing complex cognitive tasks [10]. Some studies suggest working memory performance is related to control inhibition, and can facilitate craving or relapse [11].

In relation to working memory, Bickel et al. [12] reported a component called delay discounting, which refers to the reduction of the reward received by an individual as a result of the delay in receiving it $[13,14]$. A high rate of delay discounting is correlated with a wide range of disorders and is a predictor of impairment in cognitive abilities such as decision-making and behavior control [13]. This phenomenon has a greater impact on people with addictive disorders. Improvement in working memory capacity decreases delay discounting rate [12].

Impulsivity is an example of "action without hesitation" or conduct that lacks proper evaluation of its imminent outcomes [15]. It is defined as a substrate for unplanned and rapid reactions to internal or external stimuli, without regard to their negative consequences to self or others [16]. It has a multi-dimensional structure including an emphasis on the inability to delay rewards, inhibition deficits, risk-taking and sensationseeking behavior, sensitivity to rewards, boredom, pleasure-seeking behavior, and lack of planning [5]. Pau et al. [17] found that heroin affects frontal lobes and has negative effects on attention, impulse control, and mental flexibility; however, working memory plays a fundamental and underlying role in impulse control, especially mental reasoning. Davis et al. [18] studied cognitive functions in people with

*Corresponding author: Zahra Bagheri, Institute for Cognitive Science Studies, Brain and Cognition Clinic, Tehran, Iran, Tel: +98 21 76291130; Fax: +98 21 76291130; E-mail: bagherizahra@hotmail.com

Received: October 28, 2018; Accepted: November 10, 2018; Published: November 17, 2018

Citation: Bagheri Z, Abharian PH, Armani A, Taromian F, MohammadiBytamar J, et al. (2018) Efficacy of Working Memory training on Craving and Cognitive Functioning of Opiate Users. Clin Exp Psychol 4: 202. doi 10.4172/2471-2701.1000202

Copyright: (c) 2018 Bagheri Z, et al. This is an open-access article distributed under the terms of the Creative Commons Attribution License, which permits unrestricted use, distribution, and reproduction in any medium, provided the original author and source are credited. 
a history of opioid use. They found that chronic consumers of opioids have experienced impaired impulse control.

Some neuropsychological malfunctions have also been reported among opium users. Recovered heroin users usually experience psychomotor speed disorders and attention deficits. In addition, opioid users show greater cognitive disorders compared to other patients with mental disorders [19]. Noorafkan Rouhi et al. [19] evaluated working memory performance and attention among three groups of ordinary people, opium users, and stimulant users. The results revealed that focused attention and working memory were significantly different among the three groups another study, compared executive function and working memory among current amphetamine and opioid users. The study results showed that people, who are the current or past drug user, have notable defects in spatial planning, associated learning, and pattern recognition memory.

An optimal level of cognitive function is required for psychological therapies [20]; because these therapies require patients to receive, encode, and integrate new information provided during treatment in order to become organized for treatment programs, their initiations, and implementations. In addition, addiction interventions are on a verbal basis and require extensive cognitive processing by the client in order to facilitate cognitive and behavioral changes [21-23]. Therefore, it seems reasonable to assume drug users who have cognitive defects and are involved in treatments with cognitive orientation, may face difficulties in treatment since it requires learning, and verbal and memory skills; and such skills are usually disrupted among these people [24].

The "Dual processing theory", as a cognitive model, has affected different aspects of psychology. The basic assumption in this theory is that some of our behaviors are the result of two qualitative and different types of processes: 1) relatively automated, collaborative, or impulse processing and 2) relatively controlled, symbolic, or conscious processing [25]. The model for cognitive changes resulted from addiction can be summarized as follow: cognitive defects resulted from addiction lead to strong impulsive reactions to cues for drug abuse, and compromised intellectual processing is not able to control these impulses $[10,25]$. Improving conscious processing, or weakening impulse processing can reduce the desire and impulsion an addict experiences when presented with drug use cues. It, in turn, will reduce cravings and sustains the therapy $[25,26]$.

There are three areas of human behavioral studies that investigate different aspects of the parallel processing of addictive behaviors and problems related to them. The first area deals with the moderating role of intellectual processing in impulsive behaviors. The second area investigates the changes in initial or defective cognitive impulse processing. And the third area studies the importance of interventions aimed at correcting problematic impulsive processes or empowering the person to gain control over addiction [10]. Thus, this study aims to improve working memory through computerized cognitive rehabilitation; and empower controlled processing to reduce cravings in opium dependents receiving methadone replacement treatment. It is expected that delay discounting, impulsivity, and decision-making components, known as indices of controlled processing, are improved as a result of implementing this therapeutic method.

\section{Methods}

\section{Participants}

The present study implements pretest and posttest experimental design with a control group. In this study, 30 patients with opioid abuse disorder who, in the two-month period, were referred to Addiction Medicine Clinic at Shahid Beheshti Educational Hospital of Zanjan were selected through convenience sampling. The study procedure was explained thoroughly to participants before signing the consent form. The Medical Ethics Committee of the Zanjan University of Medical Science approved the study. Then, the participants were randomly assigned to experimental and control groups in equal numbers. All 30 subjects were male and over 18 years of age with a minimum education of guidance school. All participants were receiving methadone maintenance treatment on an outpatient basis. None of them had a recent trauma to head or a mental disorder (other than addiction) in an active phase, and their addiction was not caused by a physical and/ or mental disease. All participants had perfect eyesight and hearing at the time of the study. During phase one analysis, one participant from each group was removed from the study due to not completing the questionnaire.

Treatment sessions were 30 minutes each. Before the start of each task, the therapist fully explained the method, and after ensuring the person has understood it fully, started the session. Every step of each task had three parameters: response rate, response time, and error rate, all with great importance. At the end of each task, a report of the scores for these three parameters, and the final score for each task was given to the individual.

If a person was able to pass a step successfully, difficulty degree for the next step was increased by $2 \%$; and if the person could not successfully finish a step, the difficulty level for the next step was decreased by $5 \%$ for. If someone repeatedly remained in the same step and did not improve through practice, based on the application settings for each patient, the task rolled back to the previous step in order for the participant to develop the cognitive ability required for future phases. At the end of the therapy, both control and experimental groups were reevaluated. During the treatment period, the clinic administrator was queried to assess recurrence. No recurrence reported.

\section{Materials}

All participants were evaluated before and after treatment using three questionnaires and two software applications as follows:

Wechsler working memory test software that included all subscales of Working Memory on Wechsler Memory Scale, such as forward and backward digit-span test and short-term memory span.

Two questionnaires were used to assess craving, including a desire for Drug Questionnaire of Franken et al. [27], which measures the tendency to take a drug. It includes 14 questions on a scale of zero to ten with a higher score indicating greater cravings. The questionnaire includes three factors including "desire and intention," "negative reinforcement", and "control". In order to assess the internal consistency of each factor, the Cronbach's alpha coefficients were calculated at 0.81 , 0.84 , and 0.79 for each factor, respectively. The common variance of the three factors turned out to be 0.62 . Test-retest results for each one of the factors were $0.83,0.82$, and 0.74 , respectively [27]. In the Persian version of this questionnaire, Cronbach's alpha coefficients were 0.89 , 0.79 and 0.4 , respectively [28].

The "Obsessive-Compulsive Drug Use Scale" was created to measure the properties of thoughts and obsessive practices of people dependent on alcohol. This questionnaire contains 13 questions on a scale of zero to ten with a higher score indicating greater craving. The Cronbach's alpha for internal consistency for this scale was reported 0.83 and test-retest result was 0.80 [27]. The Cronbach's alpha coefficients 
Citation: Bagheri Z, Abharian PH, Armani A, Taromian F, Mohammadi-Bytamar J, et al. (2018) Efficacy of Working Memory training on Craving and Cognitive Functioning of Opiate Users. Clin Exp Psychol 4: 202. doi: 10.4172/2471-2701.1000202

for the components in Persian version were $0.81,0.8,0.72$ and 0.45 , respectively [28].

Monetary-Choice Questionnaire (MCQ) self-report is used to evaluate the degree of delay discounting. The questionnaire contains 27 questions in which the individual needs to choose between a small but immediate monetary reward and a large but delayed monetary reward. Questions vary in term of the amount of money and the delay time. Delay discounting rate of each individual is calculated as a $\mathrm{k}$ value. High rates of delay discounting are related to impulsivity and impulsive behaviors in real life situations. The $\mathrm{k}$ value is between 0.25000 and 0.00016 . Higher scores indicate higher rates and stronger delay discounting preference for faster rewards, and hence higher levels of impulsivity.

Barratt Impulsivity Scale (BIS) contains 30 questions that are scored on a 4-point scale; those scored 64 or higher are classified as impulsive. Javid et al. [29] calculated Cronbach's alpha reliability and test-retest coefficients with an interval of two weeks for each of the impulsivity factors, which turned out to be 0.80 and 0.79 for disorganization impulsivity, 0.67 and 0.73 for motor impulsivity, 0.70 and 0.49 for cognitive impulsivity, and 0.81 and 0.77 for the whole scale.

IOWA Gambling Test software is designed to evaluate risk-taking by simulating real-life situations such as reward, punishment, and uncertainty. In this test, subjects are given four decks of cards with some fake money (e.g. \$2000). During the test, participants are asked to choose cards in an effort to lose the least amount of money and earn more money in return. Each drawer can be a reward (e.g. $\$ 1000$ for cards in decks $\mathrm{A}$ and $\mathrm{B}$ and $\$ 500$ for cards in decks $\mathrm{C}$ and $\mathrm{D}$ ) or punishment (e.g. $\$ 1000$ for cards in decks $\mathrm{A}$ and $\mathrm{B}$ and $\$ 500$ for cards in decks $\mathrm{C}$ and D). Cards in decks A and B lead to more loss, as a result, normal people are expected to avoid these decks after a few tries. The test has a total of 100 attempts; participants are not aware of this number.

\section{Results}

First, we investigated the treatment efficacy in relation to working memory. The average visual and auditory working memory prior to treatment showed no significant difference between control and study groups. The same comparison of the averages after treatment demonstrated a positive and obvious significant difference for both types of working memory (Table 1). In addition, analysis of covariance demonstrated the treatment has caused a significant difference between the two groups in terms of visual working memory and auditory working memory. Eta coefficient obtained showed that cognitive rehabilitation intervention has been able to explain the changes in great part.

The statistical comparison of means of pretest evaluations for craving, impulse control, and delay discounting showed no significant differences between the two groups. After the intervention, significant differences for all these variables were observed (Table 1). Covariance analysis demonstrated the intervention was able to create a significant difference between experimental and control groups in terms of the variables of desire to use the drug, obsessive-compulsive drug use, impulse control, and delay discounting (Table 2). Statistical analysis on decision-making showed no significant difference between the two groups before and after treatment.

\begin{tabular}{|c|c|c|c|c|c|c|}
\hline \multirow{2}{*}{ Variables } & \multirow{2}{*}{ Tests } & \multicolumn{2}{|c|}{ Control group } & \multicolumn{2}{|c|}{ Experimental group } & \multirow{2}{*}{$\mathbf{t}$} \\
\hline & & $\mathbf{M}$ & SD & $\mathbf{M}$ & SD & \\
\hline \multirow{2}{*}{ Auditory memory } & Pretest & 4.73 & 0.88 & 5.33 & 0.9 & -0.75 \\
\hline & Protest & 4.8 & 0.94 & 6.53 & 1.06 & $4.7^{* *}$ \\
\hline \multirow{2}{*}{ Visual memory } & Pretest & 5.8 & 1.26 & 5.66 & 1.49 & -0.26 \\
\hline & Protest & 5.8 & 1.26 & 7.1 & 1.24 & $2.9^{*+*}$ \\
\hline \multirow{2}{*}{ Desire for drug } & Pretest & 44.26 & 24.84 & 41.53 & 21.48 & -0.32 \\
\hline & Protest & 46.6 & 26.14 & 28.46 & 15.46 & $-2.3^{*}$ \\
\hline \multirow{2}{*}{ Obsessive-compulsive drug use } & Pretest & 53.67 & 34.8 & 59.93 & 23.19 & -0.75 \\
\hline & Protest & 68.06 & 34.8 & 27.4 & 16.87 & $-4.7^{* *}$ \\
\hline \multirow{2}{*}{ Impulse control } & Pretest & 92.2 & 13.7 & 90.88 & 15.58 & -0.25 \\
\hline & Protest & 92.98 & 16.9 & 78.06 & 11.98 & $-2.7^{* *}$ \\
\hline \multirow{2}{*}{ Delay discounting } & Pretest & 0.019 & 0.015 & 0.043 & 0.06 & 1.46 \\
\hline & Protest & 0.025 & 0.025 & 0.011 & 0.01 & $-1.97^{\star}$ \\
\hline
\end{tabular}

Table 1: Means of measured variables for 28 opium ex-addict receiving methadone treatment in two groups (with and without cognitive training).

\begin{tabular}{|c|c|c|c|c|c|c|c|}
\hline Variables & Group/Error & ss & Df & MS & $\mathbf{F}$ & Sig & $\mathbf{E}_{\mathrm{ta}}$ \\
\hline \multirow{2}{*}{ Auditory memory } & Groups & 14.05 & 1 & 14.05 & \multirow{2}{*}{$16.39^{* *}$} & \multirow{2}{*}{0.00} & \multirow{2}{*}{0.38} \\
\hline & Error & 15.23 & 27 & 0.86 & & & \\
\hline \multirow{2}{*}{ Visual memory } & Groups & 15.41 & 1 & 15.41 & \multirow{2}{*}{$36.64^{* *}$} & \multirow{2}{*}{$0 / 00$} & \multirow{2}{*}{0.55} \\
\hline & Error & 12.74 & 27 & 0.47 & & & \\
\hline \multirow{2}{*}{ Desire for drug } & Groups & 1879.12 & 1 & 1879.12 & \multirow{2}{*}{$20.69^{* *}$} & \multirow{2}{*}{00.0} & \multirow{2}{*}{0.43} \\
\hline & Error & 2452 & 27 & 90.81 & & & \\
\hline \multirow{2}{*}{ Obsessive compulsive drug use } & Groups & 8610.02 & 1 & 8610.02 & \multirow{2}{*}{$40.83^{* *}$} & \multirow{2}{*}{--} & \multirow{2}{*}{0.60} \\
\hline & Error & 5693.3 & 27 & 210.86 & & & \\
\hline \multirow{2}{*}{ Impulse control } & Groups & 1422.1 & 1 & 1422.1 & \multirow{2}{*}{$37.19^{* *}$} & \multirow{2}{*}{0.00} & \multirow{2}{*}{0.36} \\
\hline & Error & 2530.7 & 27 & 93.72 & & & \\
\hline \multirow{2}{*}{ Delay discounting } & Groups & 0.002 & 1 & 0.002 & \multirow{2}{*}{$5.49^{*}$} & \multirow{2}{*}{0.03} & \multirow{2}{*}{0.17} \\
\hline & Error & 0.01 & 27 & 0.000 & & & \\
\hline
\end{tabular}

Table 2: Covariances of measured variables for 28 opium ex-addict receiving methadone treatment in two groups (with and without cognitive training). 
Citation: Bagheri Z, Abharian PH, Armani A, Taromian F, Mohammadi-Bytamar J, et al. (2018) Efficacy of Working Memory training on Craving and Cognitive Functioning of Opiate Users. Clin Exp Psychol 4: 202. doi: 10.4172/2471-2701.1000202

Page 4 of 5

\section{Discussion}

Results of this study revealed that cognitive rehabilitation therapy of working memory could have a significant and direct effect on the reduction of opioid users' craving under methadone treatment. This finding supports the main hypothesis of the study.

According to the dual-process theory, it was expected that, by strengthening working memory, controlled processing in opioid user under methadone treatment will be boosted and as a result, their craving will diminish. Consequently, it was expected to observe positive changes in impulse control, decision making, and deferred bonus depreciation rates. Covariance analysis showed that both factors of desire to use drugs and obsessive-compulsive behavior were significantly reduced in the experimental group compared to the control group.

False and Lam [30] conducted a study and obtained comorbidity findings consistent with the present study in terms of craving. They evaluated the relative efficacy of cognitive rehabilitation therapy as an intervention for substance abuse. They randomly divided adults with substance abuse problems signed up for 12-step treatment program into two groups of 1) standard therapy plus computerized cognitive rehabilitation therapy and 2) intense attention control, including standard treatment, with computer-aided typesetting tutoring. Results showed those participated in the computerized cognitive rehabilitation therapy had higher treatment participation index and higher treatment commitment compared to the control group. In other words, the study group stayed more days in treatment compared to the control group and finished the treatment process more successfully. In follow up, a year later, significant improvements were observed on assessments related to their substance abuse and problems and they spent more days alcohol and drug-free, which represents less craving and more control over it in this group.

Hubben et al. [31] investigated the effect of strengthening working memory on restoring control over alcohol consumption behaviors. His work was based on the theory that alcohol use leads to impaired main executive functions including working memory, and that weakness of working memory leads to the sharp and more impulsive use of alcohol. Results of this study indicated that working memory training can reduce alcohol consumption and similar results were reported in the one-month follow-up study. In fact, working memory strengthening and training had also an indirect effect on impulse control. Muraven [32] concluded that two weeks of daily practice for increasing working memory capacity before quitting smoking increases the possibility of staying clean compared to the control group. In a study by Hubben et al. [31] working memory strengthening and training had an indirect effect on people's impulse control and, as a result, reduction in impulsive alcohol consumption [31]. Bickel et al. [12] reported treatment sessions focused on computerized tasks for improving working memory significantly reduced impulsivity in patients with drug addiction disorder. Additionally, in another study, Bickel et al. [12] showed that working memory training therapy sessions for people with substance use disorders can cause an apparent decline in the rate of delay discounting compared to the control group that did not receive medical treatment. The results of all the studies mentioned are consistent with the findings of the present study.

Despite what was expected based on the dual process theory, results of this study did not support the decision-making improvement hypothesis in the group receiving cognitive rehabilitation therapy compared to the control group. No significant change was observed in this component before and after treatment. Results from Dowrat et al. study demonstrated a strong relationship between working memory deficit and risky decision-making in amphetamine users with ADHD. This led to the expectation that strengthening working memory improves performance in evaluations related to risky decisionmaking [33]. It seems this faculty, as it is a complex one, needs more intervention in order for the changes to become evident or this can be because of a weakness of assessment tool.

Other limitations of the present study which presented as a weakness is a lack of follow up after the end of cognitive rehabilitation therapy. Additionally, participants were all male which precludes generalizations of the findings to the whole population of opium users. Accordingly, it is suggested studies with similar treatment approaches to be carried out with longer follow-ups, a more comprehensive and controlled population, and more accurate assessment tool especially for decision-making [34].

\section{Conclusion}

In this study cognitive training implemented at the same time with methadone treatment that is the first-line treatment for inpatients. This can ease patients' treatment procedure and improve the cognitive aspect of addiction; so better performance of patients will be seen. Given the findings of this study, investigating the efficacy of this treatment approach when referring patients to centers offering methadone treatment can be a valuable step in providing more effective treatment.

Cognitive rehabilitation therapy based on dual process theory can focus on general, non-automatic subconscious capabilities through working memory strengthening or focus on automatic and conscious capabilities such as improved attentional bias. The present study was focused on the first process and the results were expected and positive. It is suggested to conduct similar studies with improved attentional bias approach to investigate strengths and weaknesses of each approach.

\section{Funding}

Grant Agency: Zanjan University of Medical Sciences, Grant Number: Zums. rec. 1394.80

\section{References}

1. Bahari F (2013) Addiction: Counseling and treatment. Danzhe pub, Tehran.

2. Sadock I, Virginia A, Kaplan H, Rezai F (2013) Kaplan \& Sadock's Synopsis of psychiatry behavioral sciences (10thed). Arjmand company, Tehran.

3. Dehghani AF, Rostami R (2010) Effectiveness of neural feedback on craving patients dependent on opiates. Daneshvar (Raftar) Clinical Psy \& Persouality Journal of Shahed University 17: 75-84.

4. Ekhtiari H, Alam-Mehrjerdi Z, Hassani-Abharian P, Nouri M, Farnam R, et al. (2010) Examination and evaluation of carving-inductive verbal cues among Persian-speaking methamphetamine abusers. Advances in Cognitive Science 12: $28-69$

5. Mokri A, Ekhtiari H, Edalati H, Ganjgahi H, Naderi P (2008) Relationship between craving intensity and risky behaviors and impulsivity factors in different groups of opiate addicts. Iranian Journal of Psychiatry and Clinical Psychology 14: $258-268$.

6. Durazzo TC, Meyerhoff DJ, Nixon SJ (2012) A comprehensive assessment of neurocognition in middle-aged chronic cigarette smokers. Drug Alcohol Depend 122: 105-111.

7. Bozorgyan N, Zare H, Kharemin SH, Anjamrouz H (2012) Comparison of cognitive Functions of Patients with Substance Dependency and Normal People in WAIS Subscales. Armaghan Danesh 17: 469-576.

8. Simon SL, Dean AC, Cordova X, Monterosso JR, London ED (2010) Methamphetamine dependence and neuropsychological functioning: evaluating change during early abstinence. J Stud Alcohol Drugs 71: 335-344. 
Citation: Bagheri Z, Abharian PH, Armani A, Taromian F, Mohammadi-Bytamar J, et al. (2018) Efficacy of Working Memory training on Craving and Cognitive Functioning of Opiate Users. Clin Exp Psychol 4: 202. doi: 10.4172/2471-2701.1000202

9. Volkow ND, Chang L, Wang GJ, Fowler JS, Franceschi D, et al. (2000) Loss of dopamine transporters in methamphetamine abusers recovers with protracted abstinence. J Neurosci 21: 9414-9418.

10. Fadardi JS, Cox M (2009) Reversing the sequence: Reducing alcoho consumption by overcoming alcohol Attentional bias. Drug Alcohol Depend 101: 137-145.

11. Posner MI, Rothbart MK (1998) Attention, self-regulation and consciousness. Philos Trans R Soc Lond 353: 1915-1927.

12. Bickel W, Yi R, Landes R, Hill P, Baxter C (2011) Remember the Future: Working Memory Training Decreases Delay Discounting Among Stimulant Addicts. Biol Psychiatry 69: 260-265.

13. Wiers R, Stacy AW (2006) Handbook of implicit cognition and addiction. Thousand Oaks: Sage Publications.

14. Rezvanfard M, Ekhtiari M, Mokri A (2007) The relationship between personality traits and impulsivity with nicotine dependence in smokers. Advances in Cognitive Science 9: 33-49.

15. Momeni F (2010) Effectiveness of cognitive-behavior group therapy on craving reduction and quality of life promotion among opiate addiction on methadone maintenance treatment [M.A thesis]. University of social welfare and rehabilitation]. Clinical Psychology Group.

16. Dostian Y, Bahmani B, Azami Y (2013) The relationship between aggression and impulsiveness with susceptibility for addiction in male student. Rehabilitation Sciences 14: 102-109.

17. Pau CW, Lee TM, Chan SF (2002) The impact of heroin on frontal executive functions. Arch Clin Neuropsychol 17: 663-670.

18. Davis PE, Liddiard H, McMillan TM (2002) Neuropsychological deficits and opiate abuse. Drug Alcohol Depend 67: 105-108.

19. Noorafkan Roohi N, Hamidi F, Shirinabadi Farahani K (2010) Cognitive consequences of drug abuser: comparison with abuse of stimulant and opioid with regard to attention and working memory. Procedia social and behavioral science 5: 1698-1701.

20. Lundqvist $T$ (2005) Cognitive consequences of cannabis use: Comparison with abuse of stimulants and heroin with regard to attention, memory and executive functions. Pharmacol Biochem Behav 81: 319-330.

21. Li CS, Sinha R (2008) Inhibitory control and emotional stress regulation: neuroimaging evidence for frontal-limbic dysfunction in psycho-stimulant addiction. Neurosci Biobehav Rev 32: 581-597.
22. Porrino LJ, Smith HR, Nader MA, Beveridge TJ (2007) The effects of cocaine: a shifting target over the course of addiction. Neuropsychopharmacol 31: 15931600 .

23. Hersen M, Terner S (1995) Diagnostic Interview. Tehran.

24. Gregoire S, Rivalan M, Le Moine C, Dellu-Hagedorn F (2012) The synergy of working memory and inhibitory control: behavioral, pharmacological and neural functional evidences. Neurobiol Learn Mem 97: 202-212.

25. Wiers R, Gladwin T, Hofman W, Salemink E, Ridderinkhof R (2013) Cognitive bias modification and cognitive control training in addiction and related psychopathology: Mechanism, clinical perspective, and ways forward. Clinical Psychology Science 1: 192-212.

26. Hofmann W, Friese M, Roefs A (2009) Three ways to resist temptation: The independent contributions of executive attention, inhibitory control, and affect regulation to the impulse control of eating behavior. J Exp Soc Psychol 45: 431-435.

27. Franken VM, Ingmar HA Hendriks W (2002) Initial validation of two opiate craving questionnaires The Obsessive Compulsive Drug Use Scale and the Desires for Drug Questionnaire. Addict Behav 27: 675-685.

28. Hassani-Abharian P, Mokri A, Ganjgahi H, Oghabian MA, Ekhtiari H (2016) Validation for Persian version of "desire for drug questionnaire" and "obsessivecompulsive drug use scale" in heroin dependents. Arch Iran Med 19: 659-665.

29. Javid M, Mohamadi N, Rahimi CH (2011) Statistical features of Persian version of $11^{\text {th }}$ Barat Impulsivity scale. Methods and psychological models 8: 23-34.

30. False S, Lam W (2010) Computer-Assisted cognitive rehabilitation for the treatment of patients with substance use disorders: A Randomized clinical trial. Exp Clin Psychopharmacol 18: 87-98.

31. Houben K, Wiers W, Reinout J, Anita B (2011) Getting grip on drinking behavior: Training working memory to reduce alcohol abuse. Psycholo Sci 22: 968-975.

32. Muraven M (2010) Practice self-control lowers the risk of smoking lapse. Psychol Addict Behav 24: 446-452.

33. Duarte NA, Woods SP, Rooney A, Atkinson H, Grant I (2012) Working memory deficits affect risky decision-making in methamphetamine users with attentiondeficit/hyperactivity disorder. J Psychiatr Res 46: 492-499.

34. Ersche KD, Clark L, London M, Robbins TW, Sahakian BJ (2006) Profile of Executive and memory function associated with amphetamine and opiate dependence. Neuropsychopharmacol 31: 1036-1047. 